\title{
A Study on Generating Stained Glass Animation from Video Clip
}

\section{CURRENT STATUS: UNDER REVIEW}

EURASIP Journal on Image and Video Processing Springer

\section{Dongwann Kang}

Seoul National University of Science and Technology

Taemin Lee

Chung-Ang University

Yong-Hyeon Shin

Seoul National University of Science and Technology

\section{Sanghyun Seo}

Sungkyul University

sanghyun@cau.ac.krCorresponding Author

ORCiD: https://orcid.org/0000-0002-4824-3517

DOI:

10.21203/rs.3.rs-17285/v1

\section{SUBJECT AREAS}

Computer Architecture and Engineering

\section{KEYWORDS}

Non-Photorealistic Rendering, Stylization, Panoramic Image, Optical Flow 


\section{Abstract}

This paper presents a way to generate a stained glass animation from a given video input. We first obtain low-frequency components from input video frames to get rid of textures which cause oversegmentation in image segmentation. Then we segment input video volume by employing mean-shift video segmentation. The segmented regions are too large to be architecturally stable, subdivision is required. To sub-divide regions temporally coherent, we obtain the panoramic image from the segmented regions, and sub-divide them by using weighted Voronoi diagram. To render these subdivided regions as stained glass pieces, we find the best match glass piece in the real stained glass piece image database and transfer its color to that region. Finally, we generate lead came at the boundary of regions, which results in a temporally coherent stained glass animation.

Full Text

Due to technical limitations, full-text HTML conversion of this manuscript could not be completed. However, the manuscript can be downloaded and accessed as a PDF.

Figures

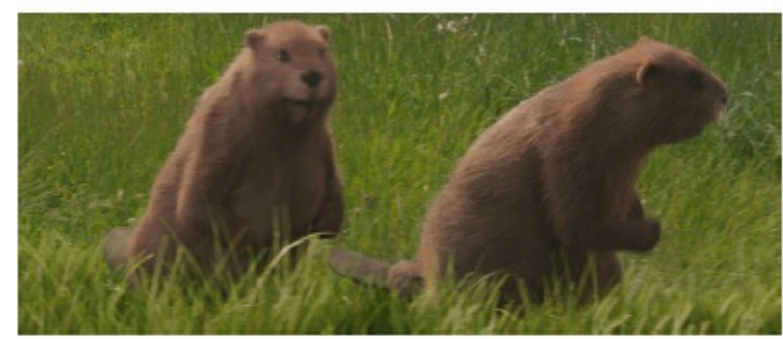

(a) Input video frame

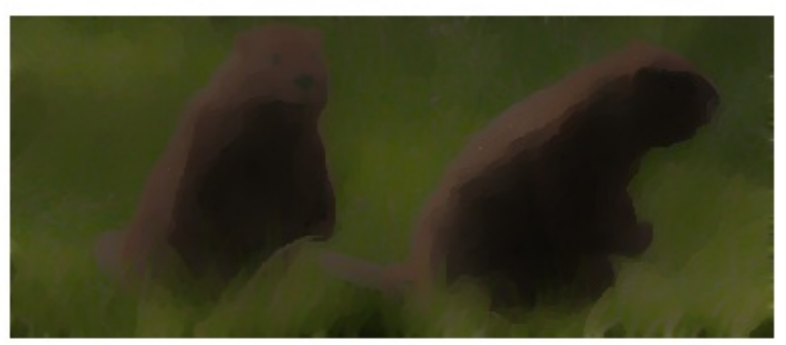

(b) Low-frequency components

Figure 1

Decomposing input video frames for getting low-frequency components. 

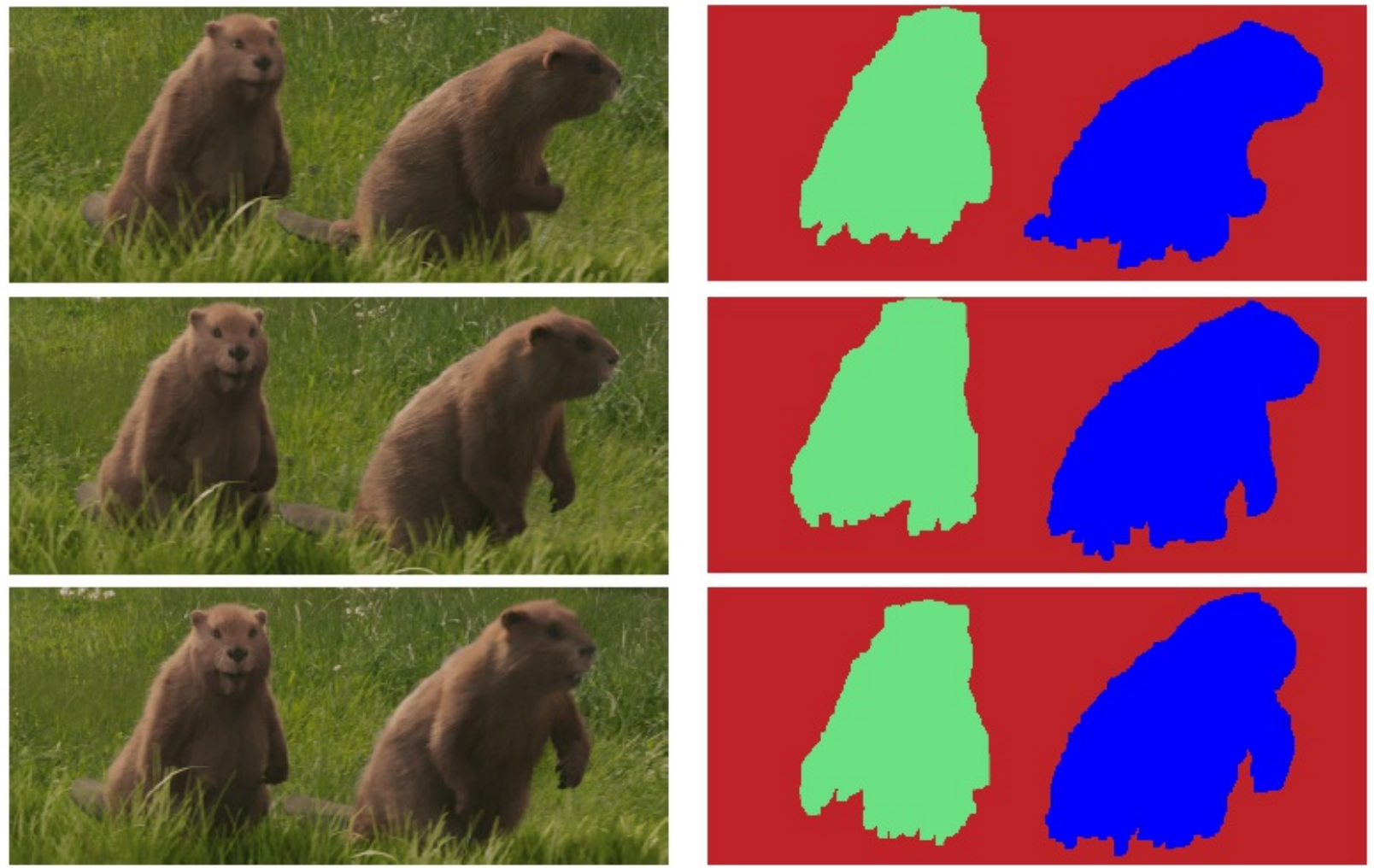

(a) Input video frames

(b) Coherently segmented regions in adjacent frames

Figure 2

Temporally coherent video segmentating using low-frequency components of video frames.

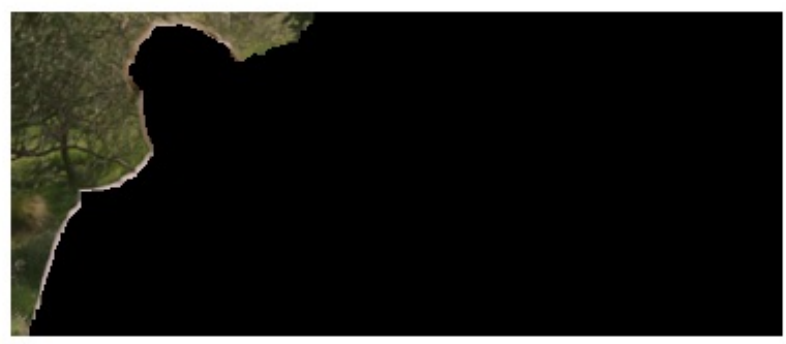

(a) Region in frame \#1

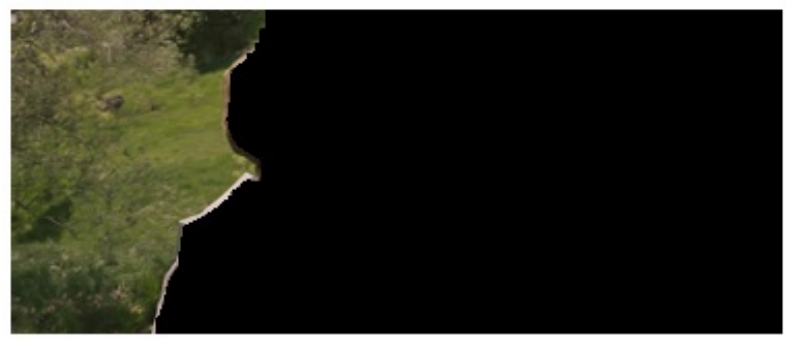

(c) Region in frame \#3

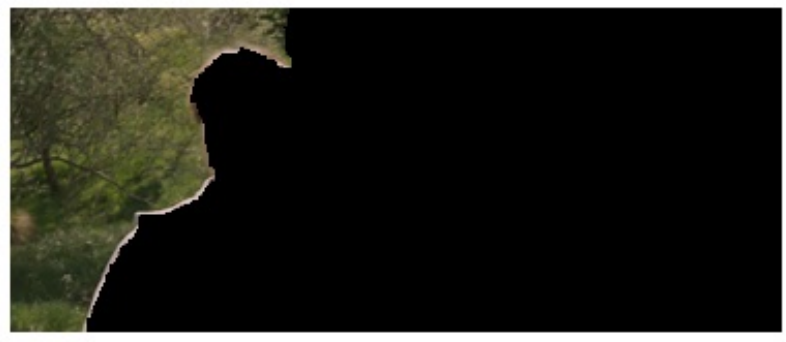

(b) Region in frame \#2

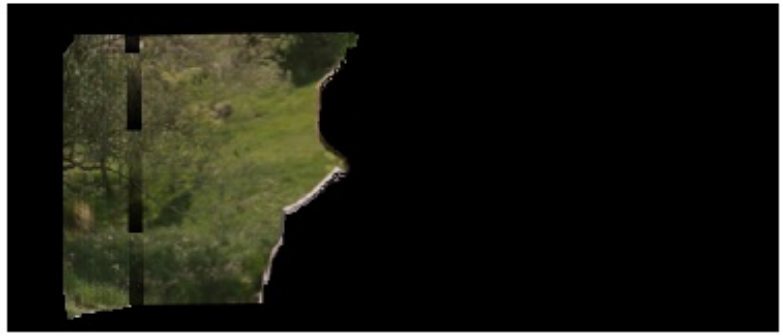

(d) Synthesized panoramic image

Figure 3

Temporally coherent video segmentating using low-frequency components of video frames. 


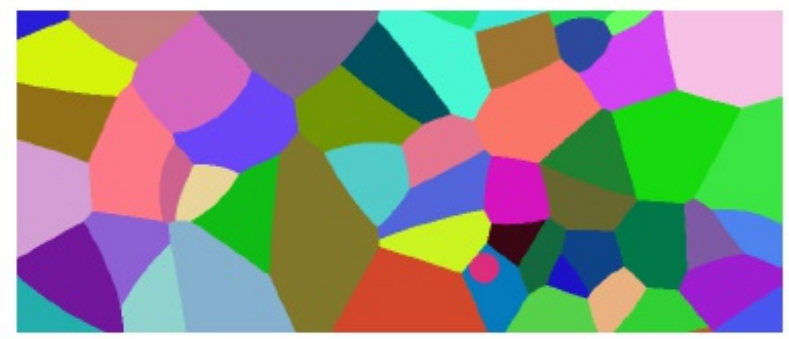

(a) Weighted Voronoi diagram on the synthesized panoramic image

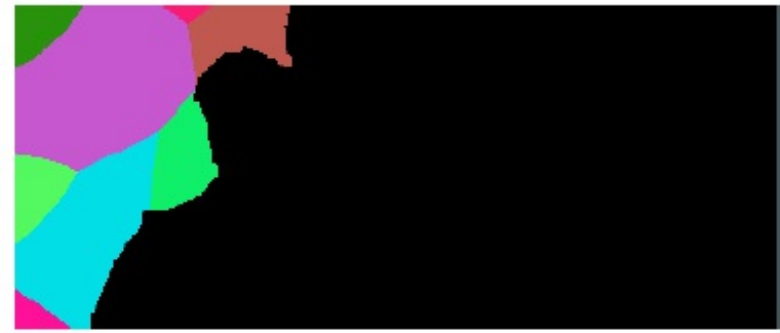

(c) Weighted Voronoi diagram on the region in frame \#2

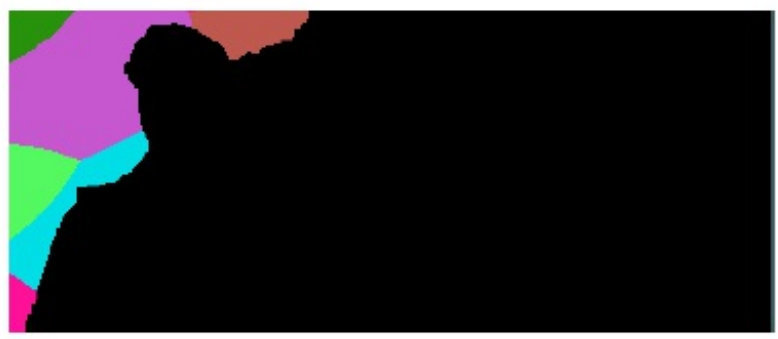

(b) Weighted Voronoi diagram on the region in frame \#1

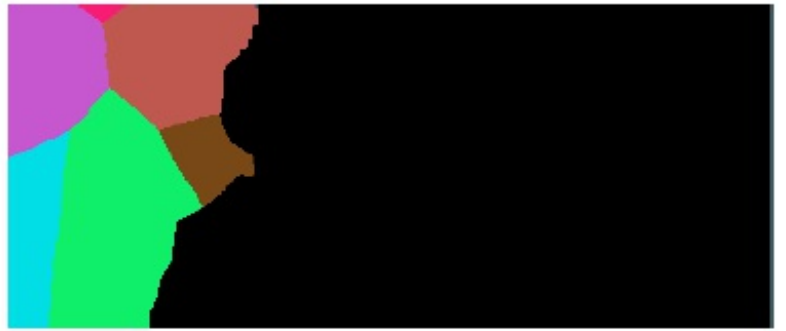

(d) Weighted Voronoi diagram on the region in frame \#3

Figure 4

Temporally coherent regions sub-divided by using weighted Voronoi diagram.
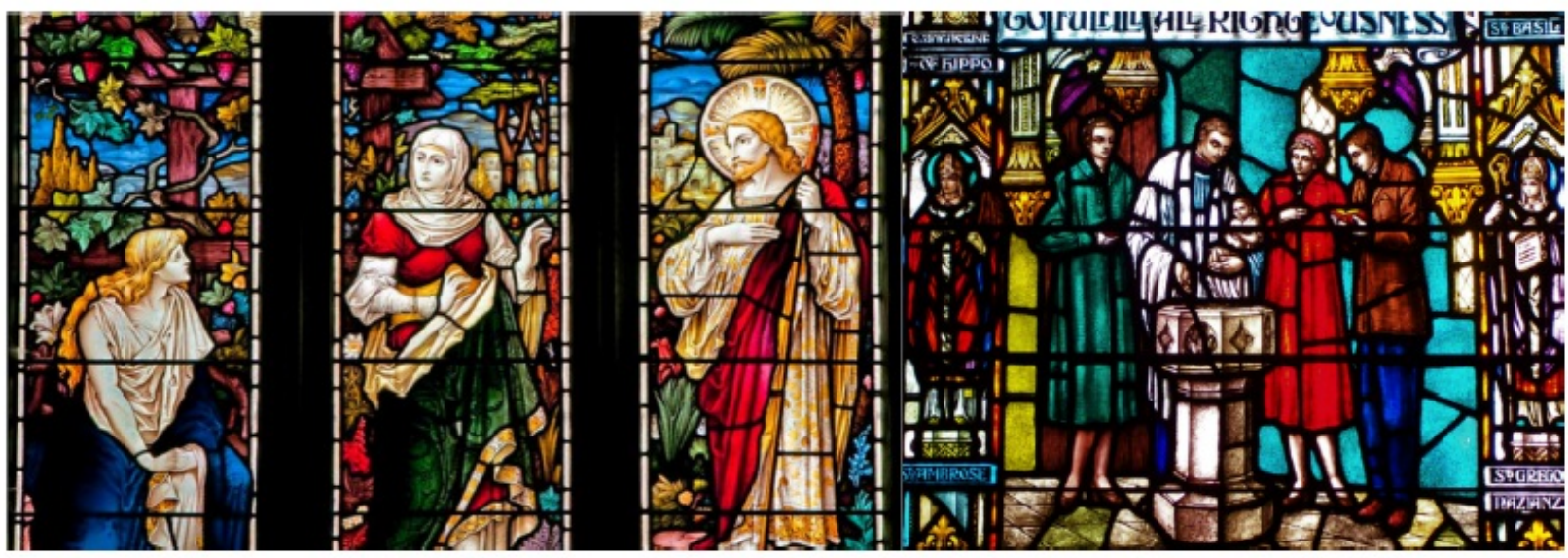

Figure 5

Sample images in our real stained glass image database.

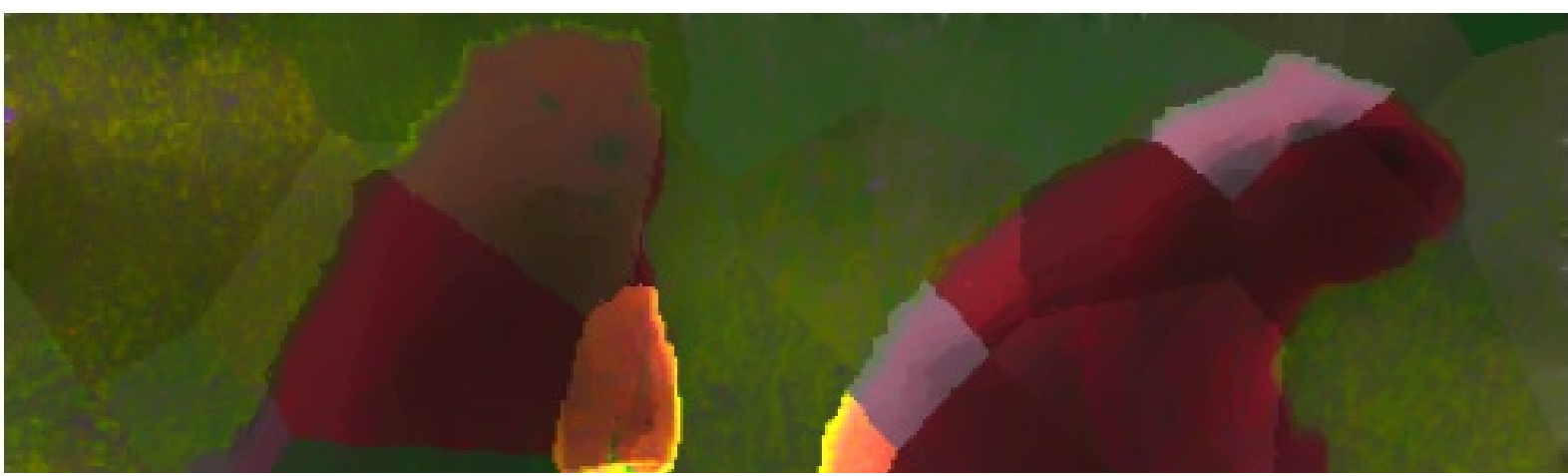




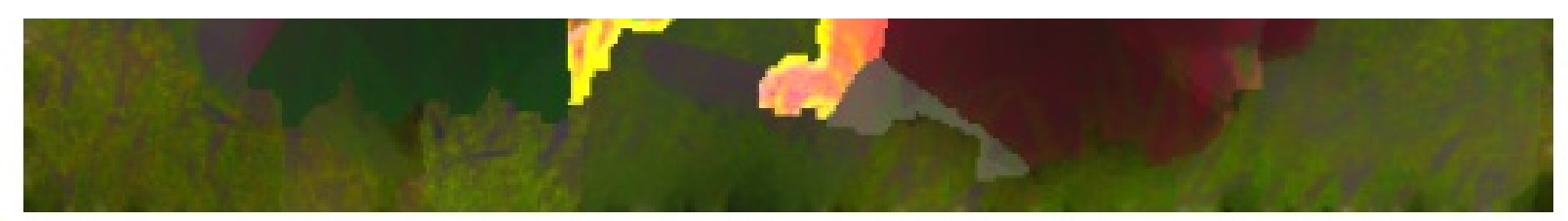

\section{(a) Color transfer}

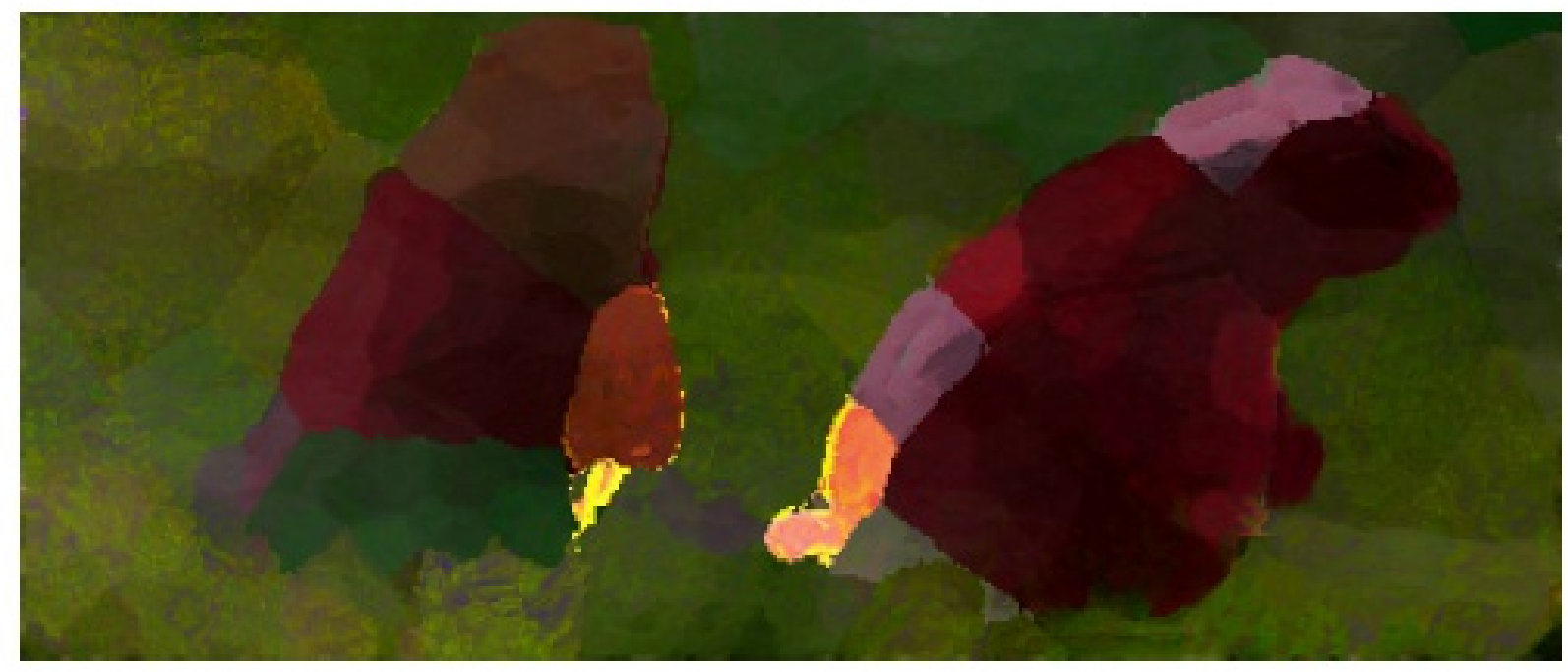

(b) The glass filter

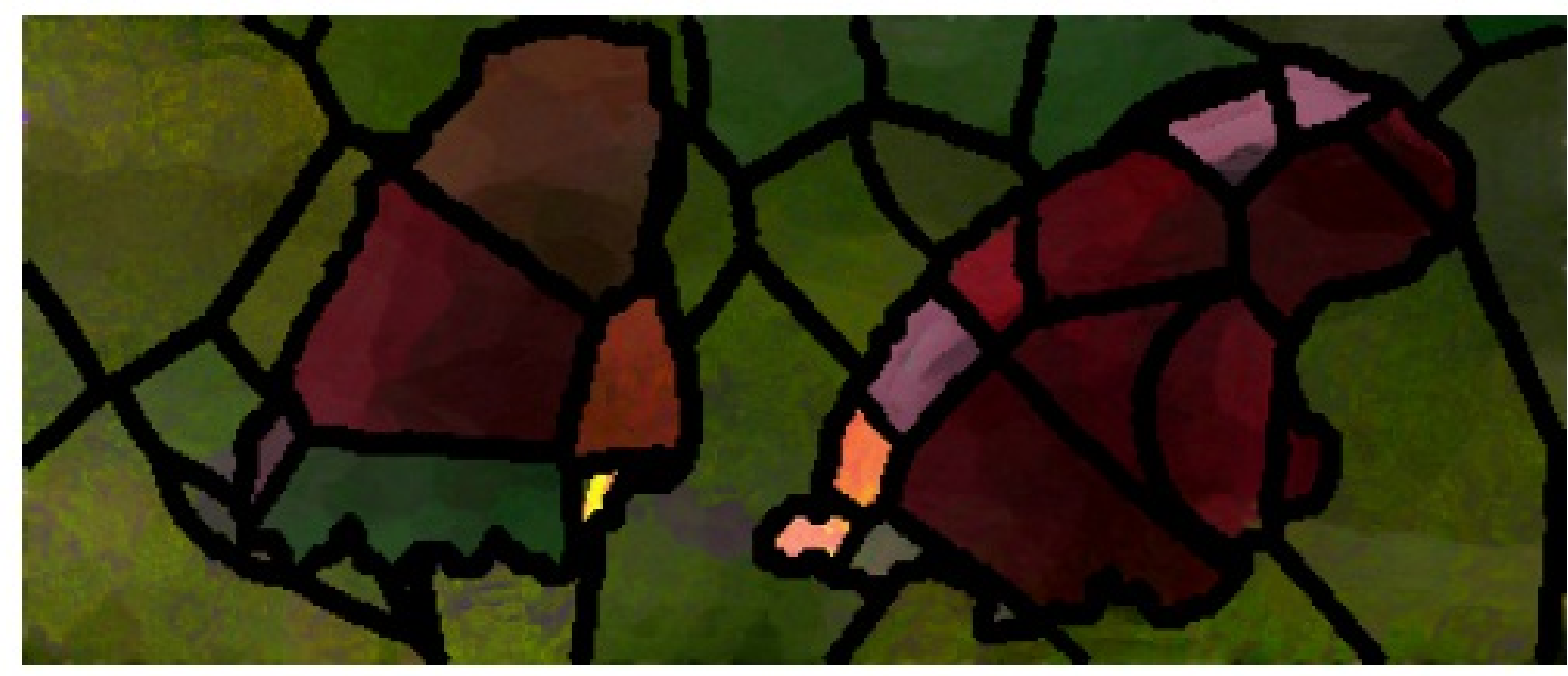

\section{(c) Lead came}

Figure 6

Stained glass rendering. 


$\begin{array}{lcc}\mathrm{D}_{\mathrm{r}} & \text { Spatial range thresholds } & 4 \\ \mathrm{D}_{\mathrm{s}} & \text { Color range threshold } & 8 \\ \mathrm{t} & \text { Time threshold } & 5\end{array}$

The minimum number of pixels of a M。 80 . segment in each frame.

Figure 7

The value of parameters used in this paper.
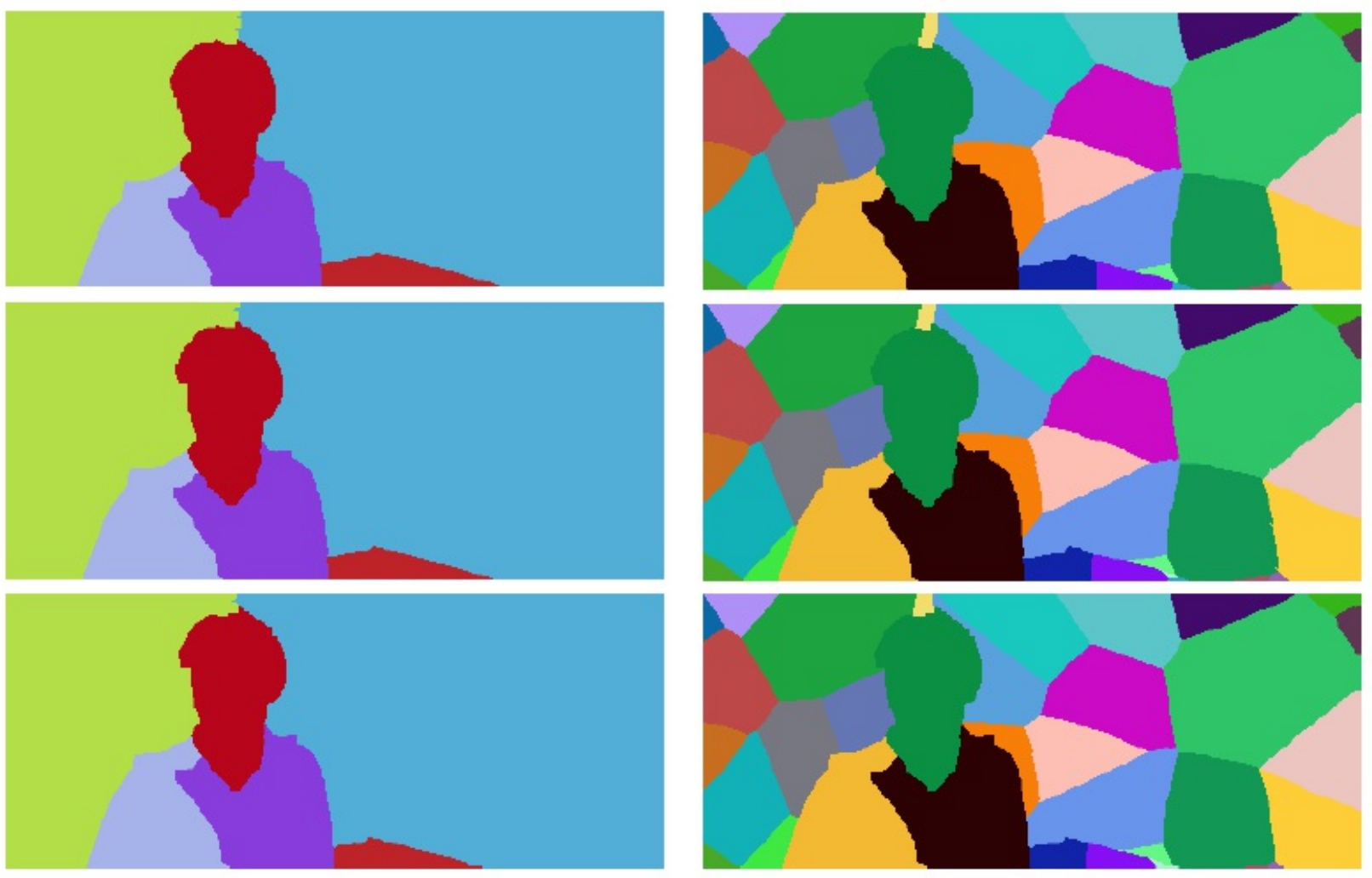

(a) Video segmentation results

(b) Artistic region sub-division results

Figure 8

Stained glass layout generation 


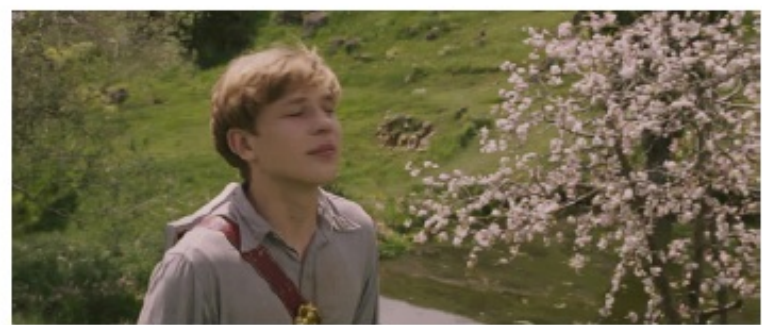

(a) An input video frame

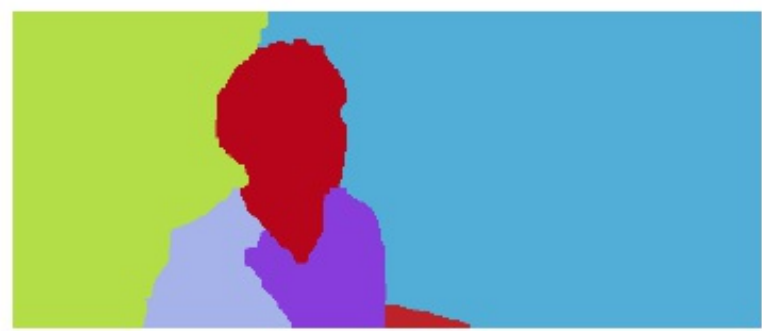

(c) Segmented regions

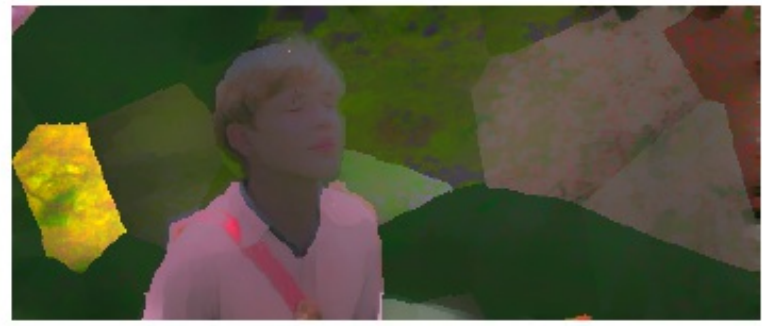

(e) Color-transfered result

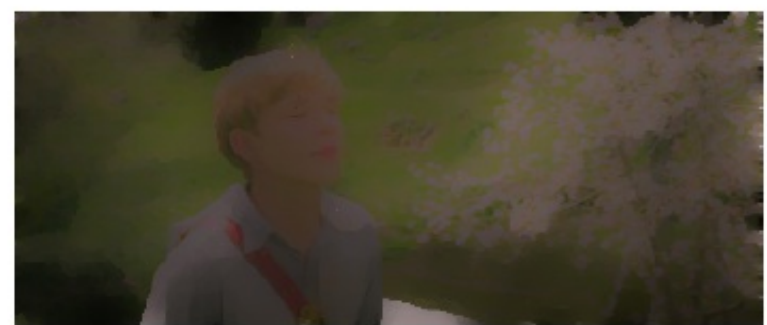

(b) The low-frequency components of input video frame

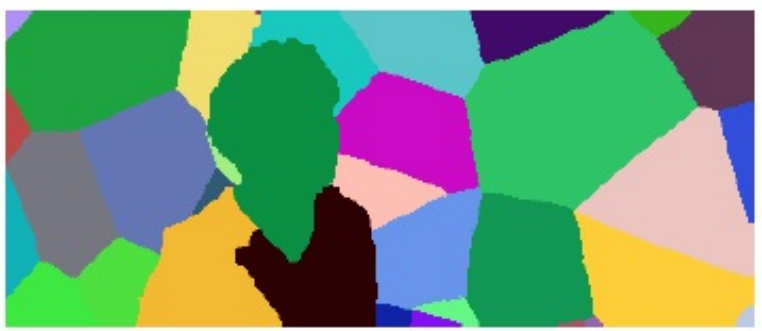

(d) Sub-divided regions

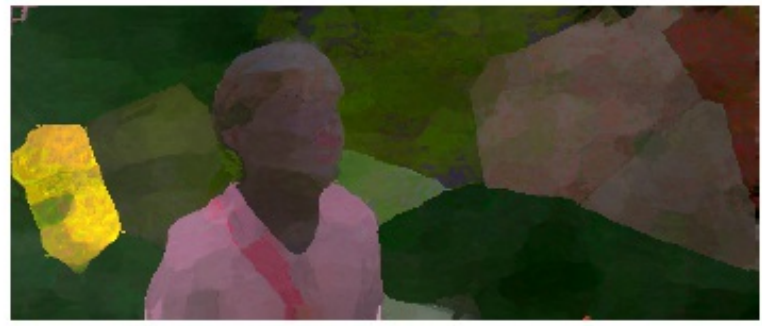

(f) Glass-filtered result

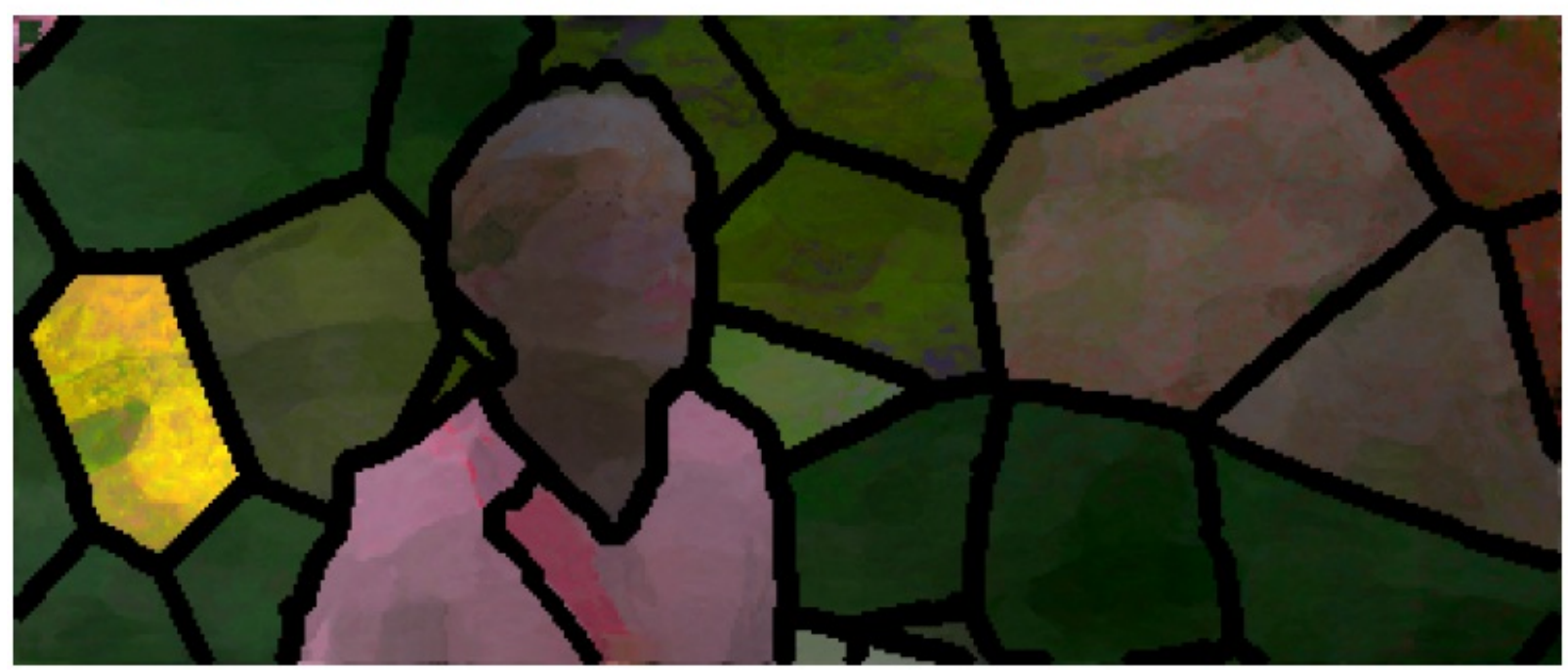

(g) Final result with lead came

Figure 9

Stained glass rendering $\&$ animation process proposed in this study. 

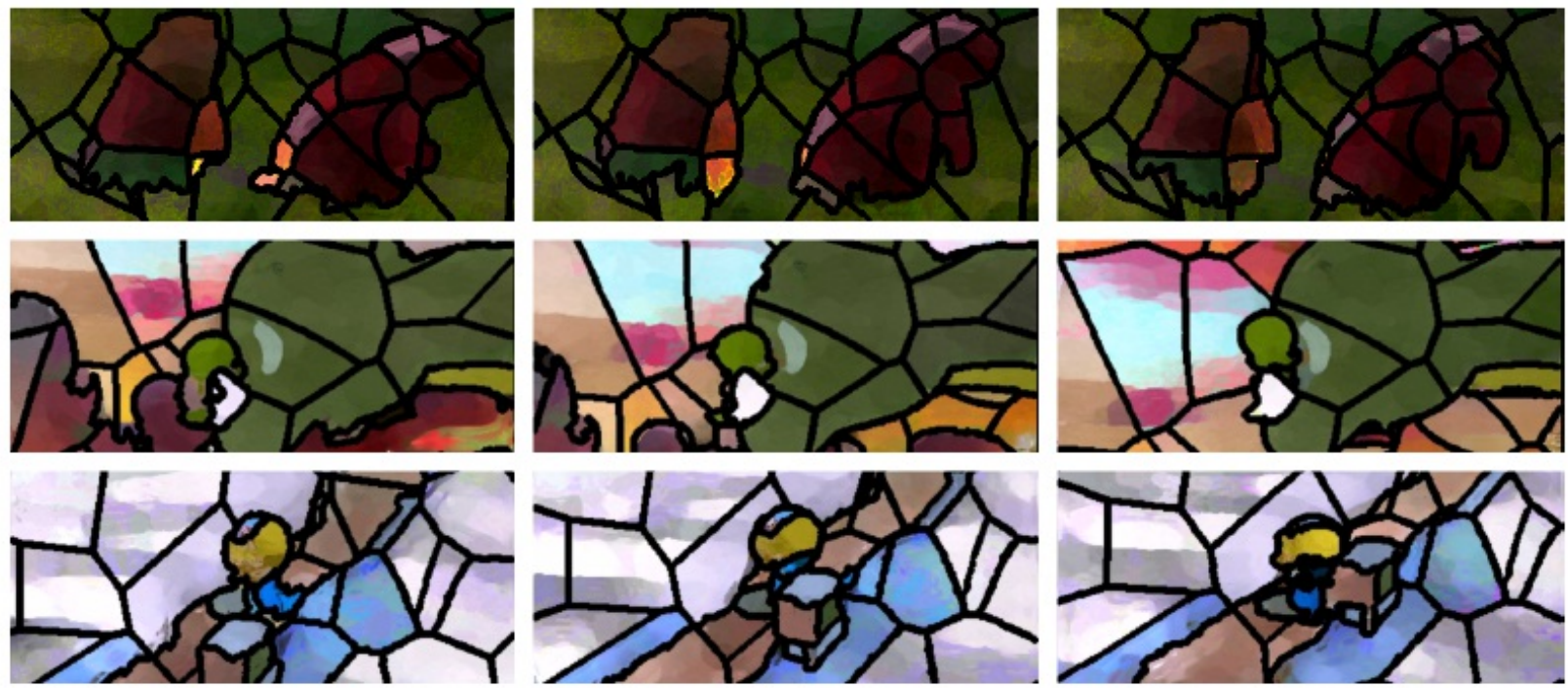

Figure 10

Various stained glass animation results.

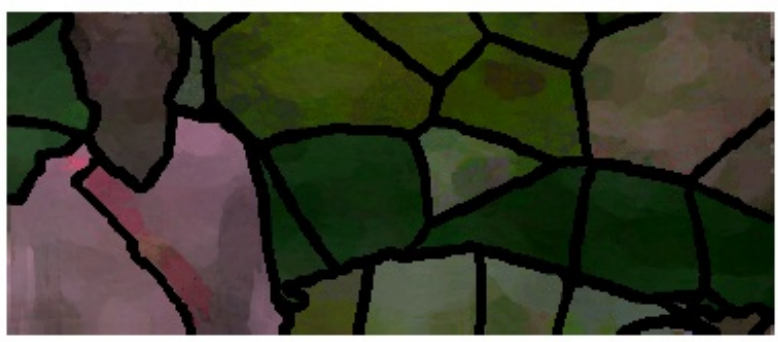

(a)

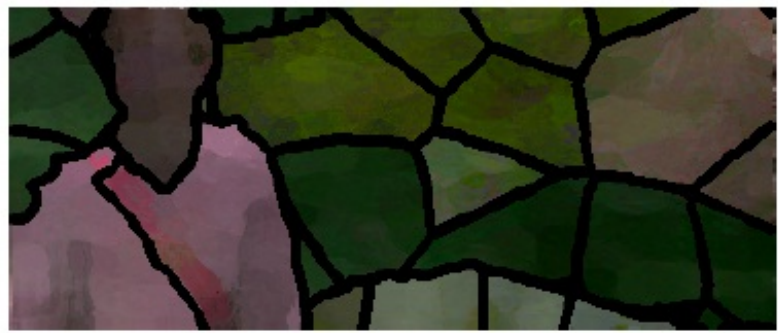

(c)

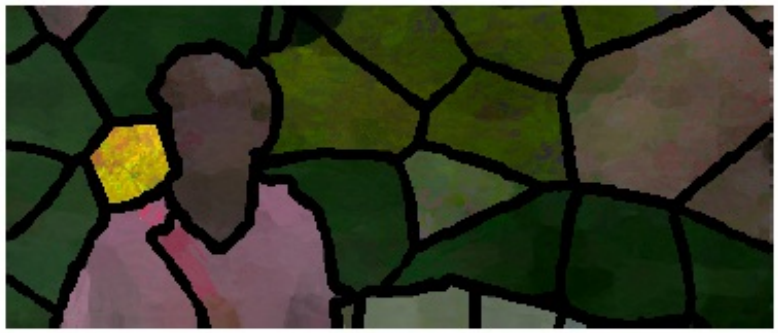

(e)

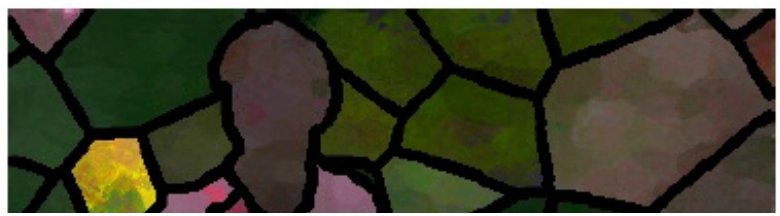

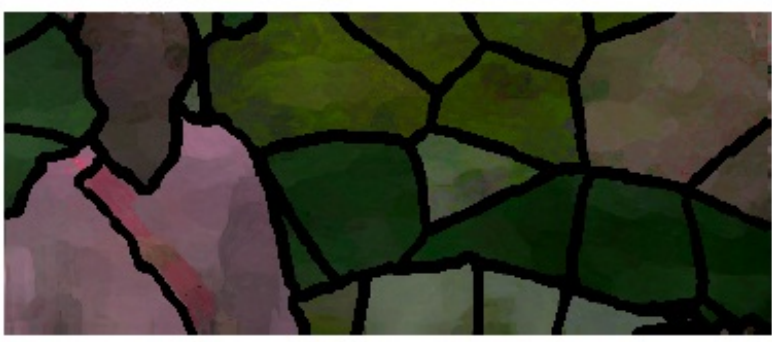

(b)

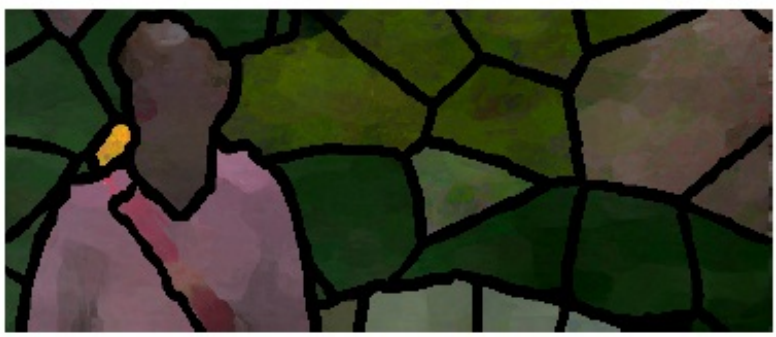

(d)

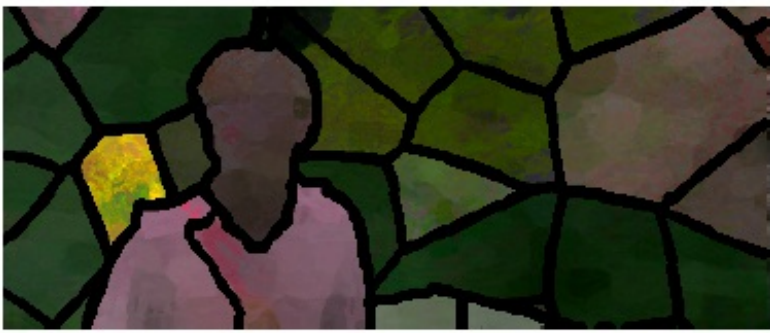

(f)

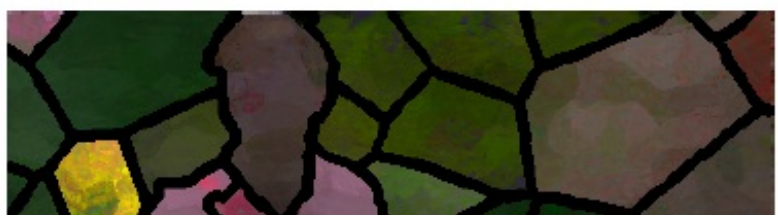




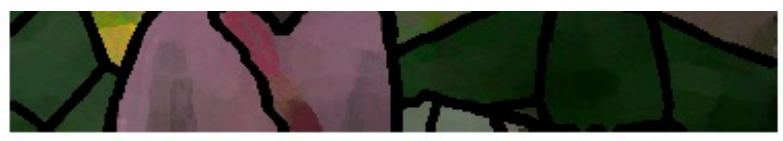

(g)

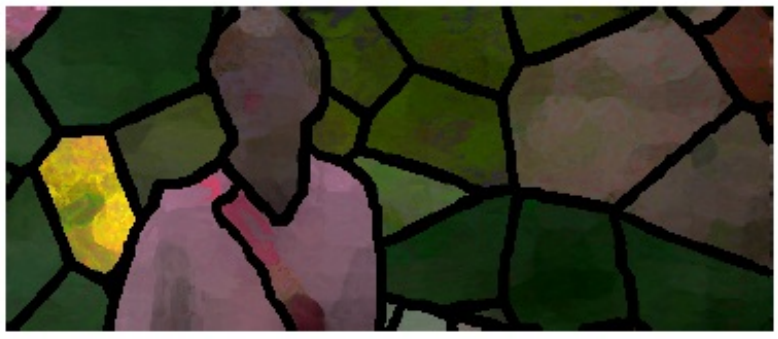

(i)

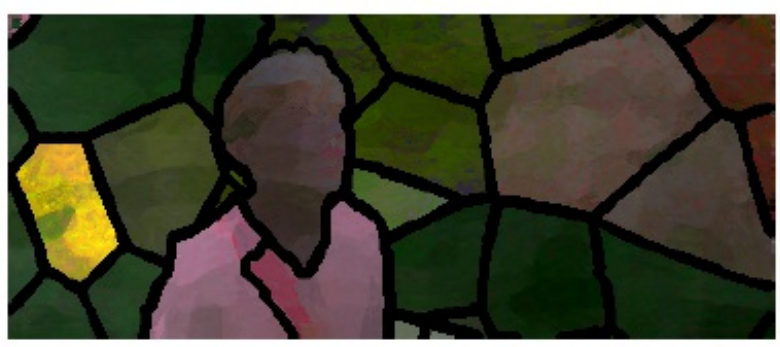

(k)

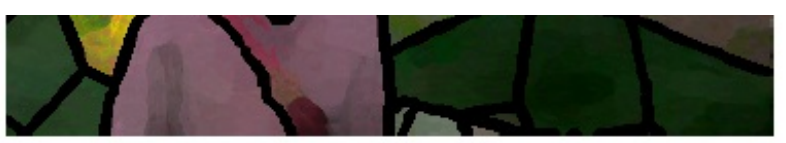

(h)

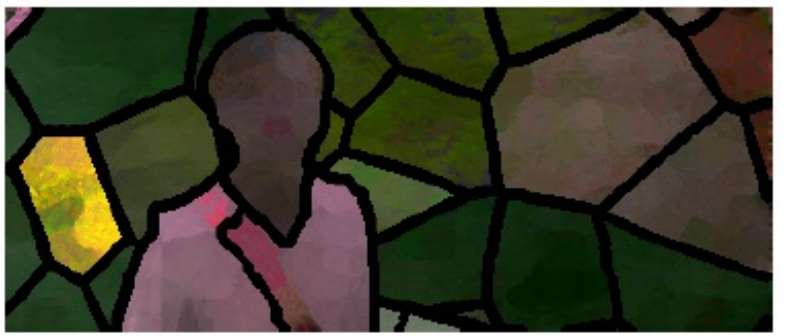

(j)

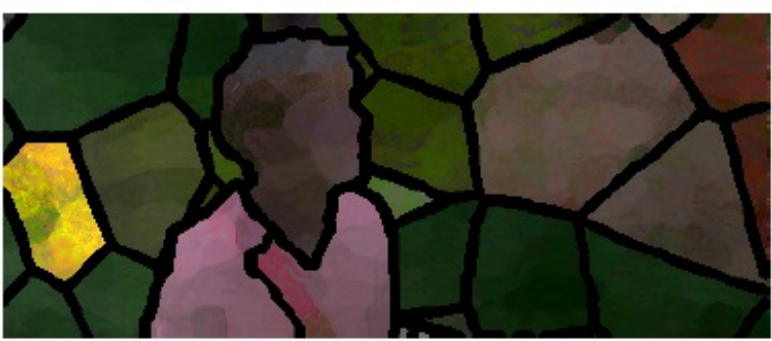

(l)

Figure 11

Stained glass animation results. 